\title{
Global existence of timelike minimal surface of general co-dimension in Minkowski space time
}

\author{
Yinxia Wang ${ }^{1 *}$ and Jingzi Liu²
}

\section{*Correspondence:}

yinxia117@126.com

ischool of Mathematics and

Information Sciences, North China

University of Water Resources and

Electric Power, Zhengzhou, 450011,

China

Full list of author information is

available at the end of the article

\begin{abstract}
In this paper, we prove that the global existence of solutions to timelike minimal surface equations having arbitrary co-dimension with slow decay initial data in two space dimensions and three space dimensions, provided that the initial value is suitably small.

MSC: $35 L 70$
\end{abstract}

Keywords: timelike minimal surface; global existence; slow decay initial value

\section{Introduction}

The theory of minimal surfaces has a long history, originating with the papers of Lagrange (1760) and the famous Plateau problem; we refer to the classical papers by Calabi [1] and by Cheng and Yau [2]. Timelike minimal submanifolds may be viewed as simple but nontrivial examples of $D$-branes, which play an important role in string theory, and the system under consideration here thus has natural generalizations motivated by string theory. The case of timelike surfaces has been investigated by several authors (see [3-5] and [6]). Huang and Kong [7] studied the motion of a relativistic torus in the Minkowski space $\mathbb{R}^{1+n}$ $(n \geq 3)$. They derived the equations for the motion of relativistic torus in the Minkowski space $\mathbb{R}^{1+n}(n \geq 3)$. This kind of equation also describes the three dimensional timelike extremal submanifolds in the Minkowski space $\mathbb{R}^{1+n}$. They showed that these equations can be reduced to a $(1+2)$ dimensional quasilinear symmetric hyperbolic system and the system possesses some interesting properties, such as nonstrict hyperbolicity, constant multiplicity of eigenvalues, linear degeneracy of all characteristic fields, and the strong null condition (see [8] and [9]). They also found and proved the interesting fact that all plane wave solutions to these equations are lightlike extremal submanifolds and vice versa, except for a type of special solution. For small initial data with compact support, the global existence problem for timelike minimal hypersurfaces has been considered by Brendle [10] and Lindblad [11].

Paul et al. [12] investigated timelike minimal submanifolds of dimension $1+n, n \geq 2$, of Minkowski spacetimes of dimension $1+n+q, q \geq 1$. The authors considered an embedding of $\mathbb{R}^{1+n}$ into Minkowski spacetime $\mathbb{R}^{1+n+q}$ given by the graph of a map $f: \mathbb{R}^{1+n} \longrightarrow \mathbb{R}^{q}$. Let Greek indices $\alpha, \beta, \ldots$ take values in $0,1, \ldots, n$ and let uppercase Latin indices $I, J, \ldots$ take values in $1, \ldots, q$. Introduce cartesian coordinates $x_{\alpha}$ on $\mathbb{R}^{1+n}$ and $x^{I}$ on $\mathbb{R}^{q}$. The induced

○2014 Wang and Liu; licensee Springer. This is an Open Access article distributed under the terms of the Creative Commons Attribution License (http://creativecommons.org/licenses/by/2.0), which permits unrestricted use, distribution, and reproduction in any medium, provided the original work is properly cited. 
metric $\mathbb{R}^{1+n}$ is

$$
h_{\alpha \beta}=\eta_{\alpha \beta}+f_{\alpha}^{I} f_{\beta}^{J} \delta_{I J}
$$

where $f_{I}=x^{I} \circ f, f^{\alpha}=\partial_{\alpha} f^{I}$ and $\eta=\operatorname{diag}(1,1, \ldots, 1)$ is the Minkowski metric. By variational principles (see [13]), they derived the Euler-Lagrange equations

$$
\partial_{\mu}\left[\sqrt{-\operatorname{det} h} h^{\mu v} f_{v}^{I}\right]=0, \quad I=1, \ldots, q
$$

Moreover for a small initial value with compact support, they also proved the global existence of classical solutions for (1.2).

In this paper, we consider (1.2) with the initial data

$$
t=0: \quad f^{I}=\varepsilon f_{0}^{I}(x), \quad f_{t}^{I}=\varepsilon f_{1}^{I}(x),
$$

where $f_{0}^{I}(x), f_{1}^{I}(x) \in C^{\infty}\left(\mathbb{R}^{n}\right)(n=2,3)$ satisfying

$$
\left|f_{0}^{I}(x)\right| \leq \frac{A}{(1+|x|)^{k}}, \quad\left|f_{1}^{I}(x)\right| \leq \frac{A}{(1+|x|)^{k+1}} \quad(k>0, I=1, \ldots, q),
$$

where $A>0$ is a constant and $\varepsilon>0$ is a small parameter. The aim of this paper is to prove that the Cauchy problem (1.2), (1.3) has a global classical solution, provided that the initial value $f_{0}^{I}(x), f_{1}^{I}(x) \in C^{\infty}\left(\mathbb{R}^{n}\right)$ is sufficiently small and satisfy $\left|f_{0}^{I}(x)\right| \leq \frac{A}{(1+|x|)^{k}},\left|f_{1}^{I}(x)\right| \leq \frac{A}{(1+|x|)^{k+1}}$ $\left(k>\frac{n}{2}, I=1, \ldots, q\right)$. We reduce the restriction on compact support of the initial data to some decay. In other words, we show the global existence of solutions to timelike minimal surface in two space dimensions and three space dimensions, provided that the initial value is suitably small.

To study (1.2), we note that (1.2) can be written in divergence form

$$
\square f^{I}=\partial_{\mu}\left[F^{\mu v} f_{v}^{I}\right]
$$

where $\square=\eta^{\mu \nu} \partial_{\mu} \partial_{v}$ is the Minkowski wave operator and $F^{\mu \nu}=\eta^{\mu \nu}-\sqrt{-\operatorname{det} h} h^{\mu \nu}$, as well as in the form

$$
H_{J L}^{\mu \nu}(\partial f) \partial_{\mu} \partial_{\nu} f^{I}=0, \quad I=1, \ldots, q,
$$

where

$$
H_{J L}^{\mu \nu}=\sqrt{-\operatorname{det} h}\left[\delta_{J L} h^{\mu \nu}-\delta_{I J} \delta_{K L}\left(h^{\mu v} h^{\alpha \beta} f_{\beta}^{K} f_{\beta}^{I}+h^{\mu \alpha} h^{\nu \beta} f_{\alpha}^{I} f_{\beta}^{K}+h^{\mu \alpha} h^{\nu \beta} f_{\alpha}^{K} f_{\beta}^{I}\right)\right] .
$$

We raise and lower Greek (intrinsic) indices using $h_{\mu \nu}$ and its inverse, while Latin (extrinsic) indices are raised and lowered using the identity $\delta_{I J}$ and its inverse. From (1.6), it follows that $H_{I J}^{\mu \nu}$ has the symmetries

$$
H_{J L}^{\mu \nu}=H_{L J}^{\mu \nu}=H_{J L}^{v \mu}
$$

Due to the symmetries, an energy estimate and local well posedness holds for the system (1.5). 
The plan of this paper is as follows. In Section 2, we cite some estimates and prove some estimates on the solution of linear wave equations. The global existence of solutions to timelike minimal surface equations with slow decay initial value in two space dimensions and three space dimensions will be proved in Section 3 and Section 4, respectively.

\section{Preliminaries}

Following Klainerman [14], we introduce a set of partial differential operators

$$
Z=\left(\left(\partial_{i}\right), i=0,1, \ldots, n ; L_{0} ;\left(\Omega_{i j}\right), 1 \leq i<j \leq n ;\left(\Omega_{0 i}\right), i=1, \ldots, n\right)
$$

where

$$
\begin{aligned}
& \partial_{0}=\frac{\partial}{\partial t}, \quad \partial_{i}=\frac{\partial}{\partial x_{i}} \quad(i=1, \ldots, n), \\
& L_{0}=t \partial_{0}+\sum_{i=1}^{n} x_{i} \partial_{i}, \\
& \Omega_{i j}=x_{i} \partial_{j}-x_{j} \partial_{i} \quad(1 \leq i<j \leq n)
\end{aligned}
$$

and

$$
\Omega_{0 i}=t \partial_{i}+x_{i} \partial_{0} \quad(i=1, \ldots, n)
$$

$Z^{\alpha}$ denotes a product of $|\alpha|$ of the vector fields (2.2), (2.3), (2.4), and (2.5). $\alpha=\left(\alpha_{1}, \ldots, \alpha_{\sigma}\right)$ is a multi-index, $|\alpha|=\alpha_{1}+\cdots+\alpha_{\sigma}, \sigma$ is the number of partial differential operators in $Z: Z=\left(Z_{1}, \ldots, Z_{\sigma}\right)$ and

$$
Z^{\alpha}=Z_{1}^{\alpha_{1}} \cdots Z_{\sigma}^{\alpha_{\sigma}}
$$

It is easy to prove Lemma 2.1 (see [15]).

Lemma 2.1 For any multi-index $\alpha=\left(\alpha_{1}, \ldots, \alpha_{\sigma}\right)$, we have

$$
\left[\square, Z^{\alpha}\right]=\sum_{|\beta| \leq|\alpha|-1} A_{\alpha \beta} Z^{\beta}
$$

and

$$
\left[\partial_{i}, Z^{\alpha}\right]=\sum_{|\beta| \leq|\alpha|-1} B_{\alpha \beta} Z^{\beta} \partial=\sum_{|\beta| \leq|\alpha|-1} \tilde{B}_{\alpha \beta} \partial Z^{\beta} \quad(i=0,1, \ldots, n)
$$

where [,] stands for the Poisson bracket, $\beta=\left(\beta_{1}, \ldots, \beta_{\sigma}\right)$ are multi-indices, $\square$ is the wave operator, $\partial=\left(\partial_{0}, \partial_{1}, \ldots, \partial_{n}\right)$ and $A_{\alpha \beta}, B_{\alpha \beta}$, and $\tilde{B}_{\alpha \beta}$ are constants.

We need the following lemma that is basically established in [16] and [17]. For completeness, the proof will also be sketched here. 
Lemma 2.2 Let $\phi_{0}(x), \phi_{1}(x) \in C^{\infty}\left(\mathbb{R}^{2}\right)$ and satisfy

$$
\left|\phi_{0}(x)\right| \leq \frac{A}{(1+|x|)^{k}}, \quad\left|\phi_{1}(x)\right| \leq \frac{A}{(1+|x|)^{k+1}} \quad(k>1) .
$$

Assume that $\phi=\phi(t, x)$ is a solution to the following Cauchy problem:

$$
\left\{\begin{array}{l}
\phi_{t t}-\triangle \phi=0, \quad x \in \mathbb{R}^{2}, t>0, \\
t=0: \quad \phi=\phi_{0}(x), \quad \phi_{t}=\phi_{1}(x), \quad x \in \mathbb{R}^{2}
\end{array}\right.
$$

Then we have

$$
|\phi(t, x)| \leq \begin{cases}\frac{C A}{\sqrt{1+t+|x|}(1+|t| x||)^{k-\frac{1}{2}}} & (|x| \geq t), \\ \frac{C A}{\sqrt{1+t+|x|} \sqrt{1+|t-| x||}} & (|x| \leq t) .\end{cases}
$$

Remark 2.1 Under the condition that

$$
\left|\phi_{0}(x)\right| \leq \frac{A}{(1+|x|)^{k+1}}, \quad\left|\phi_{1}(x)\right| \leq \frac{A}{(1+|x|)^{k+1}} \quad(k>1),
$$

Tsutaya [18] has showed that the solution of the Cauchy problem (2.9) satisfies

$$
|\phi(t, x)| \leq \frac{C A}{\sqrt{1+t+|x|} \sqrt{1+|t-| x \mid}} .
$$

Obviously, Lemma 2.2 improves the result in [18].

Proof The solution of (2.9) is given

$$
\phi(t, x)=\frac{1}{2 \pi t^{2}} \int_{|x-y| \leq t} \frac{t \phi_{0}(y)+t^{2} \phi_{1}(y)+t \nabla \phi_{0}(y) \cdot(y-x)}{\left(t^{2}-|y-x|^{2}\right)^{\frac{1}{2}}} d y .
$$

First, we make an estimate for $\left|\frac{1}{2 \pi t} \int_{|x-y| \leq t} \frac{\phi_{0}(y)}{\left(t^{2}-|y-x|^{2}\right)^{\frac{1}{2}}} d y\right|$; switching to polar coordinates, we have

$$
\begin{aligned}
\left|\frac{1}{2 \pi t} \int_{|x-y| \leq t} \frac{\phi_{0}(y)}{\left(t^{2}-|y-x|^{2}\right)^{\frac{1}{2}}} d y\right| & \frac{A}{2 \pi t} \int_{|x-y| \leq t} \frac{1}{\sqrt{t^{2}-|y-x|^{2}}(1+|y|)^{k}} d y \\
\leq & \frac{A}{2 \pi t}\left(\int_{|t-| x||}^{t+|x|} \frac{r}{(1+r)^{k}} \int_{-\varphi}^{\varphi} \frac{1}{\sqrt{t^{2}-|x|^{2}-r^{2}+2 r|x| \cos \psi}} d \psi d r\right. \\
& \left.+\chi(t-|x|) \int_{0}^{t-|x|} \frac{r}{(1+r)^{k}} \int_{-\pi}^{\pi} \frac{1}{\sqrt{t^{2}-|x|^{2}-r^{2}+2 r|x| \cos \psi}} d \psi d r\right), \\
\varphi= & \arccos \frac{|x|^{2}+r^{2}-t^{2}}{2|x| r},
\end{aligned}
$$

where $x=(|x| \cos \theta,|x| \sin \theta)$ and $y=(r \cos (\theta+\psi), r \sin (\theta+\psi))$, and $\chi$ is the characteristic function of positive numbers. 
Let $h(y)$ be a continuous function on $\mathbb{R}^{2}$ and $y=(r \cos (\theta+\psi), r \sin (\theta+\psi))$. Define

$$
H(t,|x|, r, \theta, h)= \begin{cases}\int_{-\varphi}^{\varphi} \frac{h(r, \theta+\psi)}{\sqrt{t^{2}-|x|^{2}-r^{2}+2|x| r \cos \psi}} d \psi, & \left|\frac{|x|^{2}+r^{2}-t^{2}}{2|x| r}\right| \leq 1, \\ \int_{-\pi}^{\pi} \frac{h(r, \theta+\psi)}{\sqrt{t^{2}-|x|^{2}-r^{2}+2|x| r \cos \psi}} d \psi, & \left|\frac{|x|^{2}+r^{2}-t^{2}}{2|x| r}\right| \geq 1,\end{cases}
$$

and

$$
H(t,|x|, r)=H(t,|x|, r, \theta, 1)
$$

where, as before, $\varphi$ is given by

$$
\varphi=\arccos \frac{|x|^{2}+r^{2}-t^{2}}{2|x| r}
$$

We will use the following proposition, which is proved in Kovalyov [19].

Proposition 2.1 (I) If $t \geq|x|+r$ and $\left|\frac{|x|^{2}+r^{2}-t^{2}}{2|x| r}\right| \geq 1$, then $H(t,|x|, r)$ satisfies

$$
H(t,|x|, r) \leq C \frac{\ln \left(2+\frac{r|x|}{t^{2}-(r+|x|)^{2}}\right)}{\sqrt{t^{2}-|x|^{2}-r^{2}}} \leq \frac{C}{\sqrt{t^{2}-(r+|x|)^{2}}}
$$

(II) If $t \leq|x|+r$ and $\left|\frac{|x|^{2}+r^{2}-t^{2}}{2|x| r}\right| \leq 1$, then

$$
H(t,|x|, r) \leq \frac{C}{\sqrt{r|x|}} \ln \left(2+\frac{r|x| \chi(t-|x|)}{(r+|x|)^{2}-t^{2}}\right)
$$

where $\chi$ is the characteristic function of positive numbers.

We next continue to make an estimate for (2.12); we make an estimate for the right-hand side of (2.12) by dividing into two cases.

Case 1. $|x| \geq t$.

By (2.14), we get

$$
\left|\frac{1}{2 \pi t} \int_{|x-y| \leq t} \frac{\phi_{0}(y)}{\left(t^{2}-|y-x|^{2}\right)^{\frac{1}{2}}} d y\right| \leq \frac{C A}{t \sqrt{|x|}} \int_{|x|-t}^{t+|x|} \frac{1}{(1+r)^{k-\frac{1}{2}}} d r .
$$

We subdivide into three cases again.

(i) $k>\frac{3}{2}$.

$$
\frac{C A}{t \sqrt{|x|}} \int_{|x|-t}^{t+|x|} \frac{1}{(1+r)^{k-\frac{1}{2}}} d r=\frac{C A}{t \sqrt{|x|}(1+|x|-t)^{k-\frac{3}{2}}}\left[1-\left(\frac{1+|x|-t}{1+|x|+t}\right)^{k-\frac{3}{2}}\right] .
$$

Note that

$$
1-s^{k-\frac{3}{2}} \leq C(1-s), \quad \forall 0 \leq s \leq 1
$$


and

$$
1-\frac{1+|x|-t}{1+|x|+t}=\frac{2 t}{1+|x|+t}
$$

Thus,

$$
\begin{aligned}
\left|\frac{1}{2 \pi t} \int_{|x-y| \leq t} \frac{\phi_{0}(y)}{\left(t^{2}-|y-x|^{2}\right)^{\frac{1}{2}}} d y\right| & \leq \frac{C A}{\sqrt{|x|}(1+|x|-t)^{k-\frac{3}{2}}(1+|x|+t)} \\
& \leq \frac{C A}{\sqrt{|x|+t}(1+|x|-t)^{k-\frac{1}{2}}} .
\end{aligned}
$$

(ii) $k=\frac{3}{2}$.

From (2.15), we have

$$
\begin{aligned}
& \left|\frac{1}{2 \pi t} \int_{|x-y| \leq t} \frac{\phi_{0}(y)}{\left(t^{2}-|y-x|^{2}\right)^{\frac{1}{2}}} d y\right| \\
& \quad \leq \frac{C A}{t \sqrt{|x|}} \int_{|x|-t}^{t+|x|} \frac{1}{(1+r)} d r \\
& \quad=\frac{C A}{t \sqrt{|x|}} \ln \left(1+\frac{2 t}{1+|x|-t}\right) \leq \frac{C A}{\sqrt{|x|}(1+|x|-t)} \\
& \quad \leq \frac{C A}{\sqrt{|x|+t}(1+|x|-t)} .
\end{aligned}
$$

(iii) $1<k<\frac{3}{2}$.

It follows from (2.15) that

$$
\begin{aligned}
\left|\frac{1}{2 \pi t} \int_{|x-y| \leq t} \frac{\phi_{0}(y)}{\left(t^{2}-|y-x|^{2}\right)^{\frac{1}{2}}} d y\right| & \leq \frac{C A}{t \sqrt{|x|}}\left[(1+t+|x|)^{\frac{3}{2}-k}-(1+|x|-t)^{\frac{3}{2}-k}\right] \\
& =\frac{C A}{t \sqrt{|x|}(1+|x|-t)^{k-\frac{3}{2}}}\left[\left(\frac{1+t+|x|}{1+|x|-t}\right)^{\frac{3}{2}-k}-1\right] .
\end{aligned}
$$

Note that $1<k<\frac{3}{2}$; we get

$$
\left(\frac{1+t+|x|}{1+|x|-t}\right)^{\frac{3}{2}-k}-1 \leq \frac{C t}{1+|x|-t}
$$

Hence

$$
\left|\frac{1}{2 \pi t} \int_{|x-y| \leq t} \frac{\phi_{0}(y)}{\left(t^{2}-|y-x|^{2}\right)^{\frac{1}{2}}} d y\right| \leq \frac{C A}{\sqrt{|x|+t}(1+|x|-t)^{k-\frac{1}{2}}} .
$$

In other words, if $|x| \geq t$ and $t \geq 1$, from (2.16)-(2.18), we get

$$
\left|\frac{1}{2 \pi t} \int_{|x-y| \leq t} \frac{\phi_{0}(y)}{\left(t^{2}-|y-x|^{2}\right)^{\frac{1}{2}}} d y\right| \leq \frac{C A}{\sqrt{1+|x|+t}(1+|x|-t)^{k-\frac{1}{2}}} \quad(k>1) .
$$

In what follows, we prove that (2.19) also holds if $0<t<1$ and $|x| \geq t$. In this case, we also subdivide into two cases. 
(1) $|t-| x|| \leq 1$.

By changing variables, $r=|x-y|$, we obtain

$$
\begin{aligned}
\left|\frac{1}{2 \pi t} \int_{|x-y| \leq t} \frac{\phi_{0}(y)}{\left(t^{2}-|y-x|^{2}\right)^{\frac{1}{2}}} d y\right| & \leq \frac{1}{2 \pi t} \int_{|x-y| \leq t} \frac{C A}{\left(t^{2}-|y-x|^{2}\right)^{\frac{1}{2}}(1+|y|)^{k}} d y \\
& \leq \frac{C A}{\sqrt{1+t+|x|}(1+|x|-t)^{k-\frac{1}{2}}} .
\end{aligned}
$$

(2) $|t-| x||>1$.

Note that $0<t<1$ and $|x| \geq t$, thus we obtain $|x|>t+1$. From Cases (i)-(iii), we get

$$
\left|\frac{1}{2 \pi t} \int_{|x-y| \leq t} \frac{\phi_{0}(y)}{\left(t^{2}-|y-x|^{2}\right)^{\frac{1}{2}}} d y\right| \leq \frac{C A}{\sqrt{1+t+|x|}(1+|x|-t)^{k-\frac{1}{2}}} .
$$

In other words, when $|x| \geq t$, from (2.19)-(2.21), we have

$$
\left|\frac{1}{2 \pi t} \int_{|x-y| \leq t} \frac{\phi_{0}(y)}{\left(t^{2}-|y-x|^{2}\right)^{\frac{1}{2}}} d y\right| \leq \frac{C A}{\sqrt{1+t+|x|}(1+|x|-t)^{k-\frac{1}{2}}} .
$$

Case 2. $|x| \leq t$.

From (2.12), we get

$$
\left|\frac{1}{2 \pi t} \int_{|x-y| \leq t} \frac{\phi_{0}(y)}{\left(t^{2}-|y-x|^{2}\right)^{\frac{1}{2}}} d y\right| \leq I+I I,
$$

where

$$
I=\frac{A}{2 \pi t} \int_{t-|x|}^{t+|x|} \frac{H(t,|x|, r) r}{(1+r)^{k}}
$$

and

$$
I I=\frac{A}{2 \pi t} \int_{0}^{t-|x|} \frac{H(t,|x|, r) r}{(1+r)^{k}} .
$$

In what follows, we make an estimate for $I$ and $I I$, respectively, when $t+|x| \geq 1$. It follows from (2.14) that

$$
I \leq \frac{C A}{t \sqrt{|x|}} \int_{t-|x|}^{t+|x|} \ln \left(2+\frac{|x|}{|x|+r-t}\right) \frac{1}{(1+r)^{k-\frac{1}{2}}} d r .
$$

By changing variables $\xi=|x|+r-t$,

$$
\begin{aligned}
I & \leq \frac{C A}{t \sqrt{|x|}} \int_{0}^{2|x|} \ln \left(2+\frac{|x|}{\xi}\right) \frac{1}{(1+\xi+t-|x|)^{k-\frac{1}{2}}} d \xi \\
& \leq \frac{C A}{t \sqrt{|x|}(1+t-|x|)^{k-\frac{1}{2}}} \int_{0}^{2|x|} \ln \frac{3|x|}{\xi} d \xi \\
& \leq \frac{C A}{\sqrt{1+t+|x|}(1+t-|x|)^{k-\frac{1}{2}}} .
\end{aligned}
$$


By (2.13), we get

$$
\begin{aligned}
I I & \leq \frac{C A}{t} \int_{0}^{t-|x|} \frac{1}{\sqrt{t^{2}-(|x|+r)^{2}}(1+r)^{k-1}} d r \\
& \leq \frac{C A}{t \sqrt{t+|x|}} \int_{0}^{t-|x|} \frac{1}{\sqrt{t-|x|-r}(1+r)^{k-1}} d r .
\end{aligned}
$$

Let $\rho=\sqrt{t-|x|-r}$, then

$$
\begin{aligned}
I I & \leq \frac{C A}{t \sqrt{t+|x|}} \int_{0}^{\sqrt{t-|x|}} \frac{1}{\left(1+t-|x|-\rho^{2}\right)^{k-1}} d \rho \\
& \leq \frac{C A}{t \sqrt{t+|x|}(1+t-|x|)^{\frac{k-1}{2}}} \int_{0}^{\sqrt{t-|x|}} \frac{1}{(\sqrt{1+t-|x|}-\rho)^{k-1}} d \rho .
\end{aligned}
$$

In what follows, we make estimate $I I$ by dividing into three cases.

(i) $k>2$.

$$
\begin{aligned}
I I & \leq \frac{C A}{t \sqrt{t+|x|}(1+t-|x|)^{\frac{k-1}{2}}}\left[\frac{1}{(\sqrt{1+t-|x|}-\sqrt{t-|x|})^{k-2}}-\frac{1}{(1+t-|x|)^{\frac{k-2}{2}}}\right] \\
& \leq \frac{C A}{t \sqrt{t+|x|}(1+t-|x|)^{\frac{k-1}{2}}}(\sqrt{1+t-|x|}+\sqrt{t-|x|})^{k-2} \\
& \leq \frac{C A}{\sqrt{1+t+|x|} \sqrt{1+t-|x|}} .
\end{aligned}
$$

(ii) $k=2$.

$$
I I \leq \frac{C A}{t \sqrt{t+|x|} \sqrt{1+t-|x|}} \ln \frac{\sqrt{1+t-|x|}}{\sqrt{1+t-|x|}-\sqrt{t-|x|}} \leq \frac{C A}{\sqrt{1+t+|x|} \sqrt{1+t-|x|}}
$$

(iii) $1<k<2$.

$$
\begin{aligned}
I I & \leq \frac{C A}{t \sqrt{t+|x|}(1+t-|x|)^{\frac{k-1}{2}}}\left[(1+t-|x|)^{\frac{-k+2}{2}}-(\sqrt{1+t-|x|}-\sqrt{t-|x|})^{-k+2}\right] \\
& \leq \frac{C A}{\sqrt{1+t+|x|} \sqrt{1+t-|x|}} .
\end{aligned}
$$

In other words, when $t+|x| \geq 1$, we get

$$
I I \leq \frac{C A}{\sqrt{1+t+|x|} \sqrt{1+t-|x|}}
$$

For $0<t+|x|<1$, by changing variables $r=|x-y|$, we obtain

$$
\begin{aligned}
\left|\frac{1}{2 \pi t} \int_{|x-y| \leq t} \frac{\phi_{0}(y)}{\left(t^{2}-|y-x|^{2}\right)^{\frac{1}{2}}} d y\right| & \leq \frac{1}{2 \pi t} \int_{|x-y| \leq t} \frac{C A}{\left(t^{2}-|y-x|^{2}\right)^{\frac{1}{2}}(1+|y|)^{k}} d y \\
& \leq \frac{C A}{\sqrt{1+t+|x|} \sqrt{1+t-|x|}} .
\end{aligned}
$$


Combining (2.25)-(2.27) gives

$$
\left|\frac{1}{2 \pi t} \int_{|x-y| \leq t} \frac{\phi_{0}(y)}{\left(t^{2}-|y-x|^{2}\right)^{\frac{1}{2}}} d y\right| \leq \frac{C A}{\sqrt{1+t+|x|} \sqrt{1+t-|x|}} \quad(|x| \leq t) .
$$

Thus (2.22) and (2.28) imply that

$$
\left|\frac{1}{2 \pi t} \int_{|x-y| \leq t} \frac{\phi_{0}(y)}{\left(t^{2}-|y-x|^{2}\right)^{\frac{1}{2}}} d y\right| \leq \begin{cases}\frac{C A}{\sqrt{1+t+|x|}(1+|t-| x||)^{k-\frac{1}{2}}} & (|x| \geq t), \\ \frac{C A}{\sqrt{1+t+|x|} \sqrt{1+|t-| x||}} & (|x| \leq t) .\end{cases}
$$

By Tsutaya [18], we obtain

$$
\left|\frac{1}{2 \pi} \int_{|x-y| \leq t} \frac{\phi_{1}(y)}{\left(t^{2}-|y-x|^{2}\right)^{\frac{1}{2}}} d y\right| \leq \begin{cases}\frac{C A}{\sqrt{1+t+|x|}(1+|t| x||)^{k-\frac{1}{2}}} & (|x| \geq t), \\ \frac{C A}{\sqrt{1+t+|x|} \sqrt{1+|t-| x \mid}} & (|x| \leq t),\end{cases}
$$

and

$$
\left|\frac{1}{2 \pi t} \int_{|x-y| \leq t} \frac{\nabla \phi_{0}(y) \cdot(y-x)}{\left(t^{2}-|y-x|^{2}\right)^{\frac{1}{2}}} d y\right| \leq \begin{cases}\frac{C A}{\sqrt{1+t+|x|}(1+|t-| x \mid)^{k-\frac{1}{2}}} & (|x| \geq t), \\ \frac{C A}{\sqrt{1+t+|x|} \sqrt{1+|t-| x \mid}} & (|x| \leq t) .\end{cases}
$$

Equation (2.10) follows from (2.29)-(2.31), and (2.11) immediately. Then we have completed the proof of lemma.

The following lemma plays a key role in our main results. It is basically established in [20] and [21].

Lemma 2.3 Let $\phi_{0}(x), \phi_{1}(x) \in C^{\infty}\left(\mathbb{R}^{3}\right)$ and satisfy

$$
\left|\phi_{0}(x)\right| \leq \frac{A}{(1+|x|)^{k}}, \quad\left|\phi_{1}(x)\right| \leq \frac{A}{(1+|x|)^{k+1}} \quad(k>1) .
$$

Assume that $u$ is a solution to the following Cauchy problem:

$$
\left\{\begin{array}{l}
\phi_{t t}-\triangle \phi=0, \\
t=0: \quad \phi=\phi_{0}(x), \quad \phi_{t}=\phi_{1}(x) .
\end{array}\right.
$$

Then

$$
|\phi(t, x)| \leq \frac{C A}{(1+t+|x|)(1+|t-| x||)^{k-1}} \quad(k>1) .
$$

Lemma 2.4 Let $\phi=\phi(t, x) \in C^{2}$ satisfy

$$
\square \phi+\sum_{j, k=0}^{n} \gamma^{j k}(t, x) \partial_{j} \partial_{k} \phi=F, \quad 0 \leq t \leq T
$$

and assume that $\phi$ decays to 0 at infinity. If

$$
|\gamma|=\sum\left|\gamma^{j k}\right| \leq \frac{1}{2}, \quad 0 \leq t \leq T .
$$


It follows for $0 \leq t \leq T$ that

$$
\begin{aligned}
\|\partial \phi(t, \cdot)\|_{L^{2}} \leq & 2 \exp \left(\int_{0}^{t} 2|\dot{\gamma}(\tau)| d \tau\right)\|\partial \phi(0, \cdot)\|_{L^{2}} \\
& +2 \int_{0}^{t} \exp \left(\int_{s}^{t} 2|\dot{\gamma}(\tau)| d \tau\right)\|F(s, \cdot)\|_{L^{2}} d s
\end{aligned}
$$

where $|\dot{\gamma}(t)|=\sup \left|\partial_{i} \gamma^{j k}(t, \cdot)\right|$.

For the proof of Lemma 2.4, see Klainerman [22].

Using Lemmas 2.2, 2.3 and the $L^{1}-L^{\infty}$ estimate of the linear wave equation with zero initial data, it is not difficulty to prove the following.

Lemma 2.5 Suppose that $n=2,3$. Let $\phi=\phi(t, x)$ be the solution to the Cauchy problem

$$
\left\{\begin{array}{l}
\phi_{t t}-\Delta \phi=g, \quad x \in \mathbb{R}^{n}, t>0, \\
t=0: \quad \phi=\varepsilon \phi_{0}(x), \quad \phi_{t}=\varepsilon \phi_{1}(x), \quad x \in \mathbb{R}^{n} .
\end{array}\right.
$$

Then

$$
\begin{aligned}
& (1+t)^{\frac{n-1}{2}}\|\phi(t, \cdot)\|_{L^{\infty}\left(\mathbb{R}^{n}\right)} \\
& \quad \leq C\left(\phi_{0}, \phi_{1}\right) \varepsilon+C \sum_{|I| \leq n-1} \int_{0}^{t}\left\|\left(Z^{I} g\right)(\tau, \cdot) /(1+\tau+|\cdot|)^{\frac{n-1}{2}}\right\|_{L^{1}} d \tau .
\end{aligned}
$$

By Lemmas 2.2 and 2.3, we can prove the following lemma.

Lemma 2.6 Assume that $n \geq 2$ and $\phi=\phi(t, x)$ is the solution to the Cauchy problem

$$
\left\{\begin{array}{l}
\phi_{t t}-\triangle \phi=\sum_{j=0}^{n} a_{j} \partial_{j} G_{j}, \quad x \in \mathbb{R}^{n}, t>0, \\
t=0: \quad \phi=\varepsilon \phi_{0}(x), \quad \phi_{t}=\varepsilon \phi_{1}(x), \quad x \in \mathbb{R}^{n},
\end{array}\right.
$$

where the coefficients $a_{j}(j=0, \ldots, n)$ are constants. Then we have

$$
\|\phi(t, \cdot)\|_{L^{2}} \leq C\left(\phi_{0}, \phi_{1}, G_{0}(0, \cdot)\right) m(t) \varepsilon+C \sum_{j=0}^{n} \int_{0}^{t}\left\|f_{j}(\tau, \cdot)\right\|_{L^{2}} d \tau,
$$

where

$$
m(t)= \begin{cases}\ln (2+t), & n=2, \\ 1, & n=3,\end{cases}
$$

and $C\left(\phi_{0}, \phi_{1}, G_{0}(0, \cdot)\right)$ depends on $\phi_{0}, \phi_{1}$ and $G_{0}(0, \cdot)$.

\section{Global existence in three space dimensions}

Theorem 3.1 Suppose that $f_{0}^{I}(x), f_{1}^{I}(x) \in C^{\infty}\left(\mathbb{R}^{3}\right)$ and satisfy

$$
\left|f_{0}^{I}(x)\right| \leq \frac{A}{(1+|x|)^{k}}, \quad\left|f_{1}^{I}(x)\right| \leq \frac{A}{(1+|x|)^{k+1}} \quad\left(k>\frac{3}{2}, I=1, \ldots, q\right),
$$


where $A>0$ is a constant. Then there exists $\varepsilon_{0}$ such that for $0<\varepsilon \leq \varepsilon_{0}$ the Cauchy problem (1.2), (1.3) has a global classical solution for all $t \geq 0$.

Proof The local existence argument follows from the method of Picard iteration [23] (see also [24] and [12]). In what follows, we will prove the global existence of the classical solutions by a continuous induction, or a bootstrap argument. Let $N \geq 7$, we set

$$
\begin{aligned}
& M_{1}(t)=\sum_{|\alpha| \leq N}\left\|\partial Z^{\alpha} f(t, \cdot)\right\|_{L^{2}}, \\
& M_{2}(t)=\sum_{|\alpha| \leq N}\left\|Z^{\alpha} f(t, \cdot)\right\|_{L^{2}}, \\
& N_{1}(t)=\sum_{|\alpha| \leq \frac{N+1}{2}}\left\|\partial Z^{\alpha} f(t, \cdot)\right\|_{L^{\infty}},
\end{aligned}
$$

and

$$
N_{2}(t)=\sum_{|\alpha| \leq \frac{N+1}{2}+1}\left\|Z^{\alpha} f(t, \cdot)\right\|_{L^{\infty}}
$$

To set up the bootstrap argument, we assume that there is a positive constant $K$ so that on $[0, T)$ we have the following estimates for the norms defined in (3.1):

$$
\begin{aligned}
& M_{1}(t) \leq K \varepsilon, \\
& M_{2}(t) \leq K \varepsilon, \\
& (1+t) N_{1}(t) \leq K \varepsilon,
\end{aligned}
$$

and

$$
(1+t) N_{2}(t) \leq K \varepsilon
$$

To close the bootstrap, we can prove that we can in fact choose $K$ sufficiently large and $\varepsilon$ suitably small so that the above inequalities hold independent of $T$ with $K$ replaced by $\frac{1}{2} K$.

From Lemma 2.1 and (1.5), we obtain

$$
H_{I J}^{\mu \nu} \partial_{\mu} \partial_{\nu}\left(Z^{\alpha} f^{I}\right)=\sum_{k \geq 3, \sum\left|\alpha_{i}\right| \leq|\alpha|+1} H_{I, I_{1} \cdots I_{k}, \gamma_{1} \cdots \gamma_{k}, \alpha_{1} \cdots \alpha_{k}}\left(\partial_{\gamma_{1}} Z^{\alpha_{1}} f^{I_{1}}\right) \cdots\left(\partial_{\gamma_{k}} Z^{\alpha_{k}} f^{I_{k}}\right) .
$$

It follows from Lemma 2.4 and (3.2), (3.3) for $|\alpha| \leq N$ that

$$
\begin{aligned}
M_{1}(t) & \leq C\left(\varepsilon+C \int_{0}^{t} N_{1}^{2}(\tau) M_{1}(\tau) d \tau\right) \exp \left(C \int_{0}^{t} N_{1}^{2}(\tau) d \tau\right) \\
& \leq \frac{1}{2} K \varepsilon,
\end{aligned}
$$

if $K$ is sufficiently large and $\varepsilon_{0}$ is suitably small. 
Note that $\sqrt{\operatorname{det} h} h^{\mu v}=\eta^{\mu v}+O\left(|\partial f|^{2}\right)$; from (1.4) and Lemma 2.1, we have

$$
\square\left(Z^{\alpha} f^{I}\right)=\partial_{\mu}\left[\sum_{k \geq 3, \sum\left|\alpha_{i}\right| \leq|\alpha|+1} F_{I, I_{1} \cdots I_{k}, \gamma_{1} \cdots \gamma_{k}, \alpha_{1} \cdots \alpha_{k}}^{\mu, I}\left(\partial_{\gamma_{1}} Z^{\alpha_{1}} f^{I_{1}}\right) \cdots\left(\partial_{\gamma_{k}} Z^{\alpha_{k}} f^{I_{k}}\right)\right],
$$

where again at most one of the $\alpha_{i}$ can satisfy $\left|\alpha_{i}\right|>\frac{1}{2}|\alpha|$.

Applying Lemma 2.6 to (3.5), we obtain

$$
\begin{aligned}
M_{2}(t) & \leq C \varepsilon+C \int_{0}^{t} N_{1}^{2}(t) M_{1}(t) d \tau \\
& \leq \frac{1}{2} K \varepsilon,
\end{aligned}
$$

if $K$ is sufficiently large and $\varepsilon_{0}$ is suitably small.

Since $h^{\mu v}=\eta^{\mu \nu}+O\left(|\partial f|^{2}\right)$, (3.3) may also be written as

$$
\square\left(Z^{\alpha} f^{I}\right)=\sum_{k \geq 3, \sum\left|\alpha_{i}\right| \leq|\alpha|+1} \hat{H}_{I, I_{1} \cdots I_{k}, \gamma_{1} \cdots \gamma_{k}, \alpha_{1} \cdots \alpha_{k}}\left(\partial_{\gamma_{1}} Z^{\alpha_{1}} f^{I_{1}}\right) \cdots\left(\partial_{\gamma_{k}} Z^{\alpha_{k}} f^{I_{k}}\right),
$$

where $\hat{H}=O\left(|\partial f|^{2}\right)$.

Using Lemma 2.5, (3.2), and (3.7), we get

$$
\begin{aligned}
N_{2}(t) & \leq C(1+t)^{-1}\left(\varepsilon+\int_{0}^{t} \frac{N_{1}(\tau)+N_{2}(\tau)}{1+\tau}\left(M_{1}(\tau)+M_{2}(\tau)\right)^{2} d \tau\right) \\
& \leq \frac{1}{2} K \varepsilon(1+t)^{-1}
\end{aligned}
$$

if $K$ is sufficiently large and $\varepsilon_{0}$ is suitably small.

So

$$
(1+t) N_{2}(t) \leq \frac{1}{2} K \varepsilon
$$

if $K$ is sufficiently large and $\varepsilon_{0}$ is suitably small.

From (3.1), we know that the estimate for $N_{2}(t)$ implies the desired estimate for $N_{1}(t)$. We have completed the proof of Theorem 3.1.

\section{Global existence in two space dimensions}

Theorem 4.1 Suppose that $f_{0}^{I}(x), f_{1}^{I}(x) \in C^{\infty}\left(\mathbb{R}^{2}\right)$ and satisfy

$$
\left|f_{0}^{I}(x)\right| \leq \frac{A}{(1+|x|)^{k}}, \quad\left|f_{1}^{I}(x)\right| \leq \frac{A}{(1+|x|)^{k+1}} \quad(k>1, I=1, \ldots, q),
$$

where $A>0$ is a constant. Then there exists $\varepsilon_{0}$ such that for $0<\varepsilon \leq \varepsilon_{0}$ the Cauchy problem (1.2), (1.3) has a global classical solution for all $t \geq 0$.

Proof The local existence argument follows from the method of Picard iteration [23] (see also [24] and [12]). In what follows, we will prove global existence of classical solutions by 
a continuous induction, or bootstrap argument. Let $N \geq 5$, we set

$$
\begin{aligned}
& M_{1}(t)=\sum_{|\alpha| \leq N}\left\|\partial Z^{\alpha} f(t, \cdot)\right\|_{L^{2},} \\
& M_{2}(t)=\sum_{|\alpha| \leq N}\left\|Z^{\alpha} f(t, \cdot)\right\|_{L^{2}}, \\
& N_{1}(t)=\sum_{|\alpha| \leq \frac{N+1}{2}}\left\|\partial Z^{\alpha} f(t, \cdot)\right\|_{L^{\infty}},
\end{aligned}
$$

and

$$
N_{2}(t)=\sum_{|\alpha| \leq \frac{N+1}{2}+1}\left\|Z^{\alpha} f(t, \cdot)\right\|_{L^{\infty}} .
$$

To set up the bootstrap argument, we assume that there is a positive constant $K$ so that on $[0, T)$ we have following estimates for the norms defined in (4.1),

$$
\begin{aligned}
& M_{1}(t) \leq K \varepsilon(1+t)^{\iota}, \\
& M_{2}(t) \leq K \varepsilon(1+t)^{\iota}, \\
& (1+t)^{\frac{1}{2}} N_{1}(t) \leq K \varepsilon
\end{aligned}
$$

and

$$
(1+t)^{\frac{1}{2}} N_{2}(t) \leq K \varepsilon,
$$

where $0<\iota<\frac{1}{2}$ is a fixed, arbitrary constant.

To close the bootstrap, we can prove that we can in fact choose $K$ sufficiently large and $\varepsilon$ suitably small so that the above inequalities hold independent of $T$ with $K$ replaced by $\frac{1}{2} K$.

It follows from Lemma 2.4 and (3.3) for $|\alpha| \leq N$ that

$$
\begin{aligned}
M_{1}(t) & \leq C \varepsilon \exp \left(C \int_{0}^{t} N_{1}^{2}(\tau) d \tau\right)+C \int_{0}^{t} \exp \left(C \int_{\tau}^{t} N_{1}^{2}(s) d s\right) N_{1}^{2}(\tau) M_{1}(\tau) d \tau \\
& \leq C \varepsilon \exp \left(C \int_{0}^{t} \frac{(K \varepsilon)^{2}}{1+\tau} d \tau\right)+C \int_{0}^{t} \exp \left(C \int_{\tau}^{t} \frac{(K \varepsilon)^{2}}{1+s} d s\right) \frac{(K \varepsilon)^{3}}{(1+\tau)^{1-\iota}} d \tau \\
& \leq K \varepsilon(1+t)^{\iota},
\end{aligned}
$$

if $K$ is sufficiently large and $\varepsilon_{0}$ is suitably small.

Applying Lemma 2.6 to (3.5), we obtain

$$
\begin{aligned}
M_{2}(t) & \leq C \varepsilon \ln (1+t)+C \int_{0}^{t} N_{1}^{2}(t) M_{1}(t) d \tau \\
& \leq \frac{1}{2} K \varepsilon(1+t)^{\iota},
\end{aligned}
$$

if $K$ is sufficiently large and $\varepsilon_{0}$ is suitably small. 
In what follows, we make an estimate for $N_{2}(t)$. In order to make this estimate for $N_{2}(t)$, define the following null forms:

$$
Q_{00}(u, v)=u_{t} v_{t}-\sum_{i=1}^{2} u_{i} v_{i}
$$

and

$$
Q_{i j}(u, v)=u_{i} v_{j}-u_{j} v_{i}, \quad 0 \leq i, j \leq 2, i \neq j .
$$

Let $Q$ symbolically stand for any of the full forms (4.5) and (4.6). Then

$$
Z Q(u, v)=Q(Z u, v)+Q(u, Z v)+\sum a_{i j} Q_{i j}(u, v)
$$

for some constants $a_{i j}$.

Let $Q$ be one of null form in (4.5)-(4.7), we have

$$
|Q(u, v)(t, x)| \leq C(1+t+|x|)^{-1} \sum_{|\alpha|=1}\left|Z^{\alpha} u(t, x)\right| \sum_{|\alpha|=1}\left|Z^{\alpha} v(t, x)\right| .
$$

Note that the Lagrangian associated to the volume element of the induced metric is $\sqrt{-\operatorname{det} h}$. For small $|\partial f|$, we have

$$
-\operatorname{det} h=1+\eta^{\mu v} \delta_{I J} f_{\mu}^{I} f_{v}^{J}+O\left(|\partial f|^{4}\right)=1+\delta_{I J} Q_{00}\left(f^{I}, f^{J}\right)+O\left(|\partial f|^{4}\right)
$$

and thus the Euler-Lagrange equations take the form

$$
\left(1+\delta_{K L} Q_{00}\left(f^{K}, f^{L}\right)\right) \square f^{I}=\frac{1}{2} \eta^{\mu \nu} f_{\mu}^{I} \partial_{\nu}\left[\delta_{A B} Q_{00}\left(f^{A}, f^{B}\right)\right]+O\left(\left|\partial^{2} f\right||\partial f|^{4}\right) .
$$

For small $|\partial f|$, we obtain

$$
\left(1+\delta_{K L} Q_{00}\left(f^{K}, f^{L}\right)\right)^{-1}=1+O\left(|\partial f|^{2}\right) .
$$

So we have

$$
\square f^{I}=\frac{1}{2} Q_{00}\left(f^{I}, \delta_{A B} Q_{00}\left(f^{A}, f^{B}\right)\right)+O\left(\left|\partial^{2} f\right||\partial f|^{4}\right) .
$$

By Lemma 2.1, (4.9), we have

$$
\square Z^{\alpha} f^{I}=\frac{1}{2} Q_{00}\left(Z^{\alpha_{1}} f^{I}, \delta_{A B} Q_{00}\left(Z^{\alpha_{2}} f^{A}, Z^{\alpha_{3}} f^{B}\right)\right)+O\left(\left|Z^{\beta_{1}} \partial^{2} f\right|\left|Z^{\beta_{2}} \partial f\right|^{4}\right),
$$

where $\left|\alpha_{1}\right|+\left|\beta_{2}\right| \leq|\alpha|$.

From Lemma 2.5 and (4.1), (4.2), (4.8), (4.10) when $\frac{N+1}{2}+2 \leq N$, i.e., $N \geq 5$, we get

$$
\begin{aligned}
(1+t)^{\frac{1}{2}} N_{2}(t) & \leq C \varepsilon+C \int_{0}^{t} \frac{\left(N_{1}(\tau)+N_{2}(\tau)\right)}{(1+\tau)^{\frac{3}{2}}}\left(M_{1}(\tau)+M_{2}(\tau)\right)^{2} d \tau \\
& \leq C \varepsilon+C \int_{0}^{t} K^{3} \varepsilon^{3}(1+t)^{2 \delta-2} d \tau \leq \frac{1}{2} K \varepsilon
\end{aligned}
$$

if $K$ is sufficiently large and $\varepsilon_{0}$ is suitably small and since $0<\delta<\frac{1}{2}$. 
From (4.1), we know that the estimate for $N_{2}(t)$ implies the desired estimate for $N_{1}(t)$. We have completed the proof of the theorem.

\section{Competing interests}

The authors declare that they have no competing interests.

\section{Authors' contributions}

All authors contributed to each part of this work equally and read and approved the final manuscript.

\section{Author details}

'School of Mathematics and Information Sciences, North China University of Water Resources and Electric Power, Zhengzhou, 450011, China. ${ }^{2}$ College of Electric Power, North China University of Water Resources and Electric Power, Zhengzhou, 450011, China.

Received: 13 November 2013 Accepted: 23 January 2014 Published: 07 Feb 2014

\section{References}

1. Calabi, E: Examples of Bernstein problems for some nonlinear equations. In: Proc. Sysmpos. Pure Math.. Berkely, Calif., 1968, vol. XV, pp. 223-230. Am. Math. Soc., Providence (1970)

2. Cheng, S, Yau, S: Maximal space-like hypersurfaces in the Lorentz-Minkowski spaces. Ann. Math. 104, 407-419 (1976)

3. Kong, D, Sun, Q, Zhou, Y: The equation for time-like extremal surfaces in Minkowski space $\mathbb{R}^{2+n}$. J. Math. Phys. 47, 01303 (2006)

4. Zhou, Q: The dynamics of relativistic strings moving in the Minkowski space $\mathbb{R}^{1+n}$. Commun. Math. Phys. 269, 153-174 (2007)

5. Kong, D, Zhang, Q: Solution formula and time-periodicity for the motion of relativistic strings in the Minkowski space $\mathbb{R}^{1+n}$. Phys. D, Nonlinear Phenom. 238, 902-922 (2009)

6. Milnor, T: Entire timelike minimal surfaces in $\mathbb{E}^{3,1}$. Mich. Math. J. 37, 163-177 (1990)

7. Huang, S, Kong, D: Equations for the motion of relativistic torus in the Minkowski space $\mathbb{R}^{1+n}$. J. Math. Phys. 48, $103508(2007)$

8. Christodoulou, D: Global solutions of nonlinear hyperbolic equations for small initial data. Commun. Pure Appl. Math. 39, 267-282 (1986)

9. Klainerman, S: The Null Condition and Global Existence to Nonlinear Wave Equations. Lectures in Applied Mathematics, vol. 23, pp. 293-326. Am. Math. Soc., Providence (1986)

10. Brendle, S: Hypersurfaces in Minkowski space with vanishing mean curvature. Commun. Pure Appl. Math. 28, 1249-1279 (2002)

11. Lindblad, H: A remark on global existence for small initial data of the minimal surface equation in Minkowskian space time. Proc. Am. Math. Soc. 132, 1095-1102 (2004)

12. Paul, A, Lars, A, James, I: Timelike minimal submanifolds of general co-dimension in Minkowski space time. J. Hyperbolic Differ. Equ. 3, 691-700 (2006)

13. Katsouleas, G, Maroulas, J: Variational characterizations for eigenfunctions of analytic self-adjoint operator functions. Opusc. Math. 33(2), 307-321 (2013)

14. Klainerman, S: Uniform decay estimates and the Lorentz invariance of the classical wave equations. Commun. Pure Appl. Math. 38, 321-332 (1985)

15. Li, T, Zhou, Y: Life-span of classical solution to fully nonlinear wave equations. II. Nonlinear Anal., Theory Methods Appl. 19, 833-853 (1992)

16. Wang, Y: Global existence of classical solutions to the minimal surface equation in two space dimensions with slow decay initial value. J. Math. Phys. 50, 103506 (2009)

17. Kong, D, Liu, K, Wang, Y: Life-span of classical solutions to hyperbolic geometric flow in two space variables with slow decay initial data. Commun. Partial Differ. Equ. 36, 162-184 (2011)

18. Tsutaya, K: Global existence theorem for semilinear wave equations with non-compact data in two space dimensions. J. Differ. Equ. 104, 332-360 (1993)

19. Kovalyov, M: Long-time behaviour of solutions of systems of nonlinear wave equations. Commun. Partial Differ. Equ. 12,471-501 (1987)

20. Asakura, F: Existence of a global solution to a semilinear wave equation with slowly decreasing initial data in three space dimensions. Commun. Partial Differ. Equ. 11, 1459-1487 (1986)

21. Wang, Y: Global existence of classical solutions to the minimal surface equation with slow decay initial value. Appl. Math. Comput. 216, 576-583 (2010)

22. Klainerman, S: Global existence for nonlinear wave equation. Commun. Pure Appl. Math. 33, 43-101 (1980)

23. Hörmander, L: Lectures on Nonlinear Hyperbolic Differential Equations. Mathématiques et Applications, vol. 26. Springer, Berlin (1997)

24. Sogge, C: Lecures on Nonlinear Wave Equations. Monographs in Analysis, vol. 2. International Press, Somerville (1995)

10.1186/1687-2770-2014-32

Cite this article as: Wang and Liu: Global existence of timelike minimal surface of general co-dimension in

Minkowski space time. Boundary Value Problems 2014, 2014:32 